\title{
Brasil: entre la guerra de divisas y las inversiones excesivas
}

\author{
Armando Álvares GARCIA JÚNIOR
}

\begin{abstract}
Profesor de derecho internacional público, derecho del comercio internacional y estructura económica mundial en la Universidad Internacional de la Rioja, España.

armando.alvares@unir.net
\end{abstract}

RESUMEN: La política social actual en América Latina es crecientemente inclusiva. En el caso de Brasil, eso se está logrando, de momento, mediante la reducción de las vulnerabilidades económicas externas y su sustitución por el incremento de la demanda interna. Sin embargo, la estabilidad macroeconómica también genera nuevas vulnerabilidades, pues atraen cantidades inmensas de capital externo que deprecian el dólar en el mercado interno y valorizan la moneda nacional, el real, afectando negativamente la competitividad exportadora de los productos manufacturados en el país y estimulando las importaciones de países como China, que mantiene devaluada su moneda hace muchos años. China e India son grandes compradores de materia-prima, a punto de afectar el precio de las commodities en el mercado internacional. El incremento generalizado de los precios permite aumentar la receta de los países exportadores, como Brasil, pero perpetúan un fuerte desequilibrio en las relaciones de cambio internacional y promueven la gradual desindustrialización del país.

PALABRAS-ClAVE: Economía. Finanzas. Fondo Monetario Internacional. Organización Mundial del Comercio. Brasil.

\section{Brazil: between war currency and excessive investment}

\begin{abstract}
The current social politics in Latin America is increasingly inclusive. In the case of Brazil, this is being achieved, nowadays, by means of the reduction of the external economic vulnerabilities and its substitution by the increase of the internal demand. Nevertheless, the macroeconomic stability also generates new vulnerabilities, since they attract immense quantities of external volatile capital that depreciates the dollar in the internal market and values the national currency, with negative effects on the export competitiveness of the country and stimulating the imports of countries such China, which has kept its currency devaluated for many years. China and India are important buyers of goods, affecting the price of the commodities on the international market. The widespread increase of prices allows it to increase the income of exporting countries, but maintains a strong imbalance in the relations of international exchange and promotes the gradual deindustrialisation of the country.
\end{abstract}

KEYWORDS: Economics. Finance. International Monetary Fund. World Trade Organization. Brazil.

\section{INTRODUCCIÓN. BRASIL Y LOS INVERSORES EXTRANJEROS}

Brasil, sexta economía del planeta, está atrayendo enormes inversiones extranjeras. La apertura financiera, la flexibilidad cambiaria y el control de la inflación, las altas tasas de interés, la potencialidad de su crecimiento, la estabilidad macroeconómica, política, social y normativa, la expansión y consolidación de una sociedad económicamente emergente, el incremento de la demanda interna de bienes y 
servicios, el protagonismo del país en nivel internacional, las elevadas tasas de retorno etc. son algunos de los factores que convierten Brasil en un placido oasis en medio de una horrible tormenta económica de nivel mundial.

Por otro lado, los países desarrollados sufren con la estagnación económica y las sombrías perspectivas de recuperación a corto plazo. Se añadimos el exceso de liquidez y los bajos intereses, podemos comprender fácilmente la reorientación del flujo financiero para los países emergentes, mucho más dinámicos económicamente en este momento histórico.

Sin embargo, el binomio "incremento de inversiones - devaluación competitiva de divisas" genera peligrosos efectos a medio y largo plazo al conjunto de la economía latinoamericana (especialmente en la brasileña). Explicaremos panorámicamente en este artículo los factores que sirven de base a esta afirmación.

\subsection{LA COMPETITIVIDAD} LATINOAMERICANA (CON ESPECIAL ENFOQUE A LA SITUACIÓN BRASILEÑA)

La tendencia de pérdida de competitividad en los productos manufacturados latinoamericanos tiene por base dos elementos centrales: a) el exceso de liquidez y b) la devaluación competitiva del dólar y del yuan. Ambos factores generan diversos problemas:
1) Alteración del flujo internacional del comercio. Las exportaciones latinoamericanas de productos manufacturados tienden a perder el espacio duramente conquistado en los mercados internacionales, con la sustitución gradual de la actividad productiva y exportadora por la adquisición creciente de productos importados (especialmente de China, que mantiene artificialmente devaluada su moneda para aumentar su capacidad exportadora);

2) Primarización de la economía. La gradual reducción en la actividad de las industrias en Latinoamérica genera la destrucción de puestos de trabajo en relevantes sectores secundarios y presionan los países a incrementar los productos primarios en sus líneas de exportación (en virtud del elevado precio de las commodities en el mercado internacional).

3) Valorización de la moneda nacional (real) que genera dificultades para la producción local y para el sector exportador, incrementa el valor de los factores de producción domésticos (los inputs nacionales) y estimula la 
importación (que se torna más barata), especialmente desde China., en virtud de la conversión del exceso de moneda extranjera presente en el mercado en moneda nacional, generando inflación (contenida mediante la adopción de metas inflacionarias).

El equilibrio adecuado de los niveles de intercambio comercial internacional, afectado por esos factores, repercuten en una menor incorporación de mano de obra especializada, de componentes de elevado valor añadido o tecnológico y pueden fomentar una creciente, aunque embrionaria, fuente de tensión social (parcialmente minimizada por la expansión del sector de servicios con su capacidad generadora de nuevos puestos de trabajo).

La expansión económica latinoamericana (y en especial la brasileña) es sostenible en virtud de la capacidad estatal de equilibrar crecimiento y consumo interno. La estrategia es excelente, puesto que en un cuadro de estagnación económica generalizada, con los principales mercados importadores de productos latinoamericanos aun no reactivados, la sostenibilidad basada en las exportaciones suele ser frágil, delicada $\mathrm{y}$ arriesgada.

Independiente de su suceso, es relevante considerar que la Comisión Europea ha comunicado su propuesta de suspensión (a partir de 2014) de las preferencias arancelarias concedidas para las exportaciones brasileñas. Hoy, $12 \%$ de las exportaciones brasileñas a los europeos gozan de exenciones (productos textiles, químicos, maquinas, componentes de vehículos y hasta mismo algunos productos agrícolas). Rusia, China, India y Sudáfrica (socios del BRICS) también serian excluidos.

El fin de los privilegios tiende a forzar los países a firmar acuerdos de libre comercio con la Unión Europea pero, de esa vez, para tener acceso facilitado al mercado europeo, tendrán de abrir sus mercados a los productos manufacturados.

\subsection{ASPECTOS GENERALES DE LA POLÍTICA LATINOAMERICANA EN LA HISTORIA RECIENTE}

Después del descontentamiento histórico con la derecha, incapaz de solventar los problemas de desempleo, inflación, mala distribución de la renta y exclusión social, diversos países latinoamericanos han reorientado (a partir del final de los años noventa del siglo XX) sus políticas para un modelo teóricamente más inclusivo, identificado con la izquierda (cada vez más tendente a la izquierda moderada brasileña en detrimento del radicalismo bolivariano propuesto por el presidente de Venezuela Hugo Chavez).

La democracia en la región (aunque muchas veces con rasgos populistas y demagógicos de 
sus representantes) se ha consolidado y las instituciones se han fortalecido.

En el plan continental, la concertación política regional también pasó a invocar cláusulas democráticas como su principio básico (por ejemplo, el Protocolo de Ushuaia ${ }^{1}$, de julio de 1998, para el Mercosur).

La mayor parte de los países latinoamericanos, en virtud de la reorientación política, rechazando en mayor o menor grado el neoliberalismo económico, ha optado por la adopción de una política socialmente inclusiva que objetivaba el ingreso de contingentes numéricamente expresivos de la populación más pobre en el mercado de trabajo y en el mercado de consumo, adquiriendo bienes y servicios en mayor cantidad y calidad.

La "mano invisible del mercado", mencionada por Adam Smith, no ha actuado benévolamente según la percepción de millones de personas y de muchos gobiernos, que han optado por la reincorporación de algunos sectores claves y estratégicos en la esfera pública.

Durante el apogeo del neoliberalismo en Latinoamérica, las privatizaciones y la desreglamentación económica eran habituales

$1 \quad$ La aceptación de la clausula democrática contenida en el Protocolo de Ushuaia ha sido condición para el ingreso de Venezuela en el Mercosur. Su entrada, sin embargo, aún está pendiente de la aprobación por el Congreso de Paraguay. e internacionalmente fomentadas por la política oficial del Fondo Monetario Internacional.

De hecho, el FMI ha adoptado una visión distorsionada (y posiblemente tendenciosa) de la investigación académica que resultó en el Consenso de Washington. El documento original contemplaba condiciones económicas específicas para su aplicabilidad. Sin embargo, al ser adoptado por el FMI, se ha convertido indiscriminadamente en la regla general para todos aquellos países tomadores de préstamos proporcionados por la entidad. Con la reorientación política, la izquierda latinoamericana pasó a buscar mayor equilibrio entre las fuerzas del mercado y la injerencia del gobierno en la economía.

El inicio de la crisis financiera y económica (a partir de la explosión de las denominadas hipotecas subprime en Estados Unidos en 2008) propició el incremento de sectores económicos socialmente relevantes incorporados bajo la "surveillance" gubernamental.

La reducción generalizada de tributos y el aumento real de los salarios ha fomentado un crecimiento acelerado del consumo interno, minimizando la vulnerabilidad representada por una dependencia excesiva de específicos mercados externos.

En el caso de Brasil, la reducción de tributos y el aumento salarial, acompañados de una 
mayor presencia social del Estado, ha logrado sacar millones de personas de la miseria e incorporarlas al mercado de consumo.

\subsection{BRASIL: POLÍTICAS DE REDUCCIÓN DE VULNERABILIDADES EXTERNAS E INCREMENTO DE LA DEMANDA INTERNA}

Las medidas gubernamentales adoptadas en Brasil en los últimos años han logrado reducir un grave problema, consistente en una expansión económica domestica inapropiada a la sostenibilidad del desarrollo económico y del bien estar social a largo plazo, en virtud de su acoplamiento a una demanda interna insuficiente de los agentes microeconómicos.

La estrategia gubernamental de desarrollo económico y sustentación política, en nivel domestico, básicamente se ha hecho, en esa primera etapa, con el fomento de la actividad microeconómica. El incremento de la demanda interna por parte del consumidor final implicaría por su parte en el incremento de las ventas de los minoristas, cuyos productos eran solicitados a los mayoristas y esos, por su vez, demandados a las industrias. Ese aumento de la demanda interna ha implicado, en cierta medida, en una desvinculación de la excesiva dependencia externa en términos de demanda (vulnerabilidad macroeconómica externa).
La ampliación y consolidación de nuevos socios comerciales hace años permitió al gobierno brasileño, por ejemplo, disminuir proporcionalmente la concentración de los productos nacionales destinados al mercado estadounidense. El objetivo del gobierno es tornarse un global player en toda la regla.

\subsection{LA $\quad$ ESTABILIDAD MACROECONÓMICA GENERA NUEVAS VULNERABILIDADES}

En Brasil existe una variante de la denominada "enfermedad holandesa" concerniente a los problemas generados en el país en virtud de su propio suceso. Otros países latinoamericanos, como Perú, pueden presentar la misma tendencia a medio plazo.

Básicamente, los Bancos Centrales necesitan realizar operaciones de swap de cambio reverso, equivalente a la compra de dólares en el mercado futuro, para intentar contener la presión de valorización de la moneda local en virtud de la devaluación del dólar en el mercado interno (generada por su ingreso excesivo en el país).

¿Por qué ingresa tanto capital extranjero en ciertos países? La respuesta presenta muchas variables: a) población con poder adquisitivo creciente, b) estabilidad macroeconómica, c) estabilidad jurídica, d) gobierno democrático (los gobiernos autoritarios suelen adoptar medidas drásticas e imprevisibles), e) perspectiva de crecimiento del PIB, f) 
perspectiva de incremento de la renta per capita, g) buena actividad de los agentes microeconómicos, h) carencias o perspectivas de crecimiento de sectores económicos determinados, i) incentivos gubernamentales, j) alta tasa de retorno financiero, k) tipo de interés.

Una moneda se valora, entre otras razones, porque el Banco Central necesita retirar del mercado el exceso de moneda extranjera. En el caso brasileño, el "real" ha sido la moneda que más se ha valorado en la última década. Las compras casi diarias realizadas por el Banco Central para retirar del mercado el volumen excesivo de dólares invertidos en el país han incrementado el valor del real y ha convertido las reservas monetarias brasileñas en las más elevadas de su historia, con más de 350 mil millones de dólares.

Cuanto mayor la estabilidad y la seguridad macroeconómica, incluyendo la reserva estratégica, más capital externo atrae ese país para su mercado interno. En tiempos de crisis, países económicamente estables son como un oasis en el desierto.

Los elevados intereses practicados por los países latinoamericanos, si por un lado son utilizados como medidas de política económica para contener la inflación, por otro, sirven como una farola luminosa que atrae capital extranjero, muchas veces de naturaleza volátil.
Con el incremento de la importación por la valorización del moneda, las industrias nacionales se retraen y las materias primas pasan a ganar cada vez mayor espacio en las exportaciones del país, manteniendo un perverso desequilibrio de los medios de intercambio entre los países. Como ejemplo, algunas grandes empresas brasileñas han empezado a trasladar sus cadenas productivas para India o Nicaragua, por ejemplo.

Lamentablemente, la expansión de la demanda interna para compensar la retracción externa en tiempos de crisis no puede ser completamente efectiva, aunque ayude. Ciertos productos manufacturados con elevado valor añadido no pueden ser consumidos internamente, pues la demanda interna es claramente insuficiente para compensar financieramente la producción.

En este sentido, los millones de toneladas de soja, los aviones etc. producidos en Brasil, siguen siendo artículos del comercio exterior de naturaleza esencialmente extra-continental. Los vecinos latinoamericanos no podrían compensar satisfactoriamente la retracción en ese campo.

Por otro lado, las compras gubernamentales, utilizadas como política de incentivo y fomento para la producción de ciertos sectores estratégicos, encuentran fuerte resistencia por parte de otros países (ese ha sido uno de los 
motivos del impase del Área de Libre Comercio de las Américas, ALCA).

\subsection{EXPORTACIONES DE PRODUCTOS PRIMARIOS EN LATINOAMÉRICA. EL PAPEL DE CHINA}

El gradual crecimiento de los productos primarios en la pauta exportadora de varios países latinoamericanos está generando un desarrollo asimétrico y en condiciones desfavorables de intercambio internacional.

El precio elevado de las commodities en el mercado internacional asegura importantes ingresos de capital al país exportador pero su precio está, actualmente, estrechamente relacionado a la colosal demanda de China e India.

China depende de la importación de materias primas (especialmente minerales, petróleo y derivados) para llevar a cabo su proyecto nacional de industrialización creciente, base de su sostenibilidad social. En un contexto político de partido único (el partido comunista), es absolutamente imperioso mantener bajo control las tensiones sociales.

La continuidad del crecimiento del país evita que la tensión social atinja niveles que escapen al control del partido comunista. En China, las políticas de desarrollo industrial y de infraestructura cumplen esa finalidad. Si la economía china deja de crecer al ritmo de los últimos años, el reflejo sobre la importación de hierro y otros productos minerales será inmenso.

Alimentos, minerales y petróleo configuran elementos que, en este momento, corresponden a intereses de política estratégica para la mayor parte de los gobiernos en el mundo.

Los alimentos, en virtud de la escalada de precios y presiones inflacionistas, presentan un perfil social importante que testan continuamente a los gobiernos, democráticos (o no democráticos, como es el caso de China).

La tendencia agresiva de adquisición de tierras cultivables en otros países para producción e importación de alimentos por gobiernos de países con poca posibilidad de expansión agrícola (por limites de espacio, calidad del suelo, condiciones climáticas o crecimiento/densidad demográfica), o por empresas privadas relacionadas con el gobierno, sirve para tranquilizar la populación y evitar eventuales protestos que afecten a la estabilidad del país.

En relación a los minerales, existe una tendencia creciente de los gobiernos en evitar la exportación de ciertos minerales (denominados en su conjunto como "tierras raras", cuya exploración es completamente 
dominada por China), especialmente después de la realización de estudios científicos que ponen en relieve su elevado poder estratégico para el empleo en las nuevas tecnologías: componentes de circuitos, conductores, discos duros de ordenadores, motores de aviones, equipamientos médicos etc.

Así que la exportación de minerales sigue centrada especialmente en el hierro, el cobre etc. utilizados en las construcciones y obras que están siendo llevadas a cabo por países como China e India.

Por último, el petróleo se opone a la tendencia generalizada de sustitución del combustible fósil por combustibles renovables y mucho más limpios (la Unión Europea tiene un importante plan para el 2020 y otro para el 2050).

Relativamente a los productos primarios, se percibe un gradual contraste y una inconveniente división entre Sudamérica (que se está expandiendo mientras India y China absorban sus exportaciones de hierro, cobre, soja, café, carbón, petróleo, trigo, aves, carnes, azúcar etc.) y los demás países de la región.

Los patrones de comercio exterior y de inversiones en Sudamérica son más intensos, diversificados y dinámicos (a pesar de los constantes impasses en el Mercosur y de los reiterados obstáculos impuestos por el gobierno argentino a los productos brasileños) que en otras partes del continente americano (hay que observar que gran parte de las riquezas actualmente explotadas en América están ubicadas en Sudamérica).

No hay muchos países ricos en minerales o con una agricultura pujante en América Central o en las islas del Caribe: café, banana, azúcar y carne bovina son producidos allí pero en escala insuficiente para sostener un boom de desarrollo económico y social.

\subsection{EE.UU Y LATINOAMÉRICA. LOS ACUERDOS BILATERALES}

Muchos de los países de Centroamérica y Caribe exportan bienes primarios $\mathrm{o}$ manufacturas de bajo valor añadido y se mantienen por el turismo y por el dinero procedente de las remesas de sus nacionales residentes en el extranjero (valores decrecientes en virtud del regreso de miles de personas a sus respectivos países de origen desde que la crisis golpeó con fuerza a los países europeos y a los Estados Unidos de América).

Una subárea americana está se libertando muy gradualmente de la omnipresente hegemonía estadounidense pero su prosperidad está estrechamente relacionada con la expansión económica de India y China, compradores de sus productos primarios en larga escala. La otra subárea americana está cada vez más integrada a los Estados Unidos (mayoritariamente) y al Canadá (en menor 
escala) y sus economías están fuertemente vinculadas a esos dos mercados.

El gobierno estadounidense ha renovado diversos acuerdos bilaterales cuando el proyecto de construcción del Área de Libre Comercio de las Américas (ALCA) naufragó en 1995 por la resistencia del gobierno brasileño en aceptar la forma de negociación impulsada por el gobierno estadounidense.

México, Chile, Colombia y Perú han firmado en 2011 y Colombia en 2012 (después de la clausura de la VI Cumbre de las Américas) acuerdos de libre comercio con EE.UU, avanzando la integración económica de las Américas en una dirección rechazada por Brasil a partir de 2003. Cada acuerdo ofrece facilidades de acceso al mercado más importante del mundo.

Los cuatro países también han firmado en 2011 un tratado internacional bautizado como "Acuerdo del Pacífico" y que constituirá un mercado mayor que el brasileño. Por ser miembro del Mercosur, formalmente una unión aduanera, Brasil no puede negociar con México un acuerdo de libre comercio. Los gobiernos brasileño y mexicano tienen firmado acuerdos más limitados.

PRIMARIZACIÓN
POSICIONAMIENTOS COMUNES

En Brasil, la exportación de commodities supera la de productos manufacturados. Casi mitad de ellas está concentrada en mineral de hierro, petróleo en bruto, complejo de soja, azúcar (bruto y refinado), carnes, acero y celulosa.

Mientras los precios de las commodities (incluyendo las agrícolas) sigan elevados, los países exportadores de materias primas seguirán exportando porque se benefician del crecimiento de la receta. Más dinero ingresado, mayor posibilidad de tocar adecuadamente la economía, si la corrupción permite.

La idea de adoptar posiciones unificadas para aumentar la presión negociadora en foros internacionales que discuten los controles de precios de las commodities ha encontrado obstáculos hasta mismo dentro de los BRICS (Brasil, Rusia, India, China y, desde abril de 2011, Sudáfrica). A propósito, Sudáfrica ya es socio de Brasil en el foro IBAS (India, Brasil, Sudáfrica), un proyecto de especial interés del gobierno brasileño.

India y Rusia son grandes importadores de alimentos, al paso que Brasil es un grande exportador. Los primeros simpatizan con la idea de un mayor control de los precios al paso que Brasil, por supuesto, rechaza la idea de regular y controlar la volatilidad de la cotización internacional de productos básicos como alimentos y petróleo. 
La acomodación de interés divergentes culminó con la "Declaración de Sanya" en que se afirmó el control de la volatilidad de los precios de las commodities por medio del aumento de la producción (deseada por Brasil) y por intermedio de una mejor regulación del mercado de derivativos (deseado por China). El tema ganará cada vez más espacio, ya que la excesiva volatilidad pone en riesgo la recuperación de la economía global.

Para los países exportadores de materiasprimas, como los sudamericanos, el precio más elevado en los mercados internacionales se traduce como mayor ingreso de capitales en el país, lo que les interesa. Sin embargo, el precio elevado de las commodities genera inflación en todo el mundo y, como vimos, produce la desindustrialización de los países exportadores. Desde luego, el problema de la inestabilidad de precios en el mercado internacional se ha agravado cuando las materias primas empezaron a ser tratadas en los mercados especulativos como activos financieros.

Los gobiernos sudamericanos temen una regulación jurídica que permita el control de los precios y de los mercados de commodities agrícolas. Diplomáticamente objetivan una posición unificada dentro del Grupo de los Veinte (centrada en el aumento de la producción como factor de reducción de los precios internacionales). El problema es que Brasil y Argentina son los únicos países de la región que integran el G-20.

El resultado final de las diversas discusiones ha sido el del control sobre las operaciones especulativas (incluyendo especialmente los derivativos), sobre los productos agrícolas.

\section{REFERÊNCIAS BIBLIOGRÁFICAS}

BRASIL. Ministerio de Asuntos Exteriores de Brasil. Documentos diversos. Brasilia 2009, 2010, 2011 y 2012.

BRASIL. Ministerio de Asuntos Exteriores de Brasil. Discursos seleccionados. Brasilia 2009, 2010, 2011 y 2012.

BRASIL. Senado Federal de Brasil.

Documentación. Brasilia. 2009, 2010, 2011 , 2012.

BRASIL. Senado Federal. Reseña de leyes. Brasilia. 2000-2012.

GARCIA JR. Armando Alvares. Manual de Estructura Económica Mundial. UNIR, Madrid-Logroño, 2012 (en prensa).

GARCIA JR. Armando Alvares. Direito Internacional Público Moderno. Editora Aduaneiras, São Paulo, 2012 (en prensa).

GARCIA JR. Armando Alvares. Direito Internacional - Questões Atuais $2^{\mathrm{a}}$ edit.. Aduaneiras São Paulo, 2005

GARCIA JR., Armando Alvares. Contratos Dolarizados no Direito Brasileiro. Ltr, São Paulo, 2000. 